\title{
Análise espacial das amputações de membros em um estado do Nordeste
}

\author{
Spatial analysis of limb amputations in a Northeastern state \\ Análisis espacial de amputaciones de extremidades en un estado del Noreste
}

Recebido: 12/09/2021 | Revisado: 19/09/2021 | Aceito: 20/09/2021 | Publicado: 21/09/2021

Regina Nunes da Silva
ORCID: https://orcid.org/0000-0001-5957-076X
E-mail: renusill@yahoo.com.br
Universidade Estadual de Ciências da Saúde
Rosário de Fátima Alves de Albuquerque
ORCID: https://orcid.org/0000-0002-6227-9700
E-mail: rosarioalbuquerque @ hotmail.com
Paulyanne Karlla Araújo Magalhães
ORCID: https://orcid.org/0000-0002-6731-6515
Instituto Federal de Alagoas, Brasil
E-mail: paulyanne.ifal@gmail.com
Ewerton Amorim dos Santos
Universidade Estadual de Ciências da Saúde de Alagoas, Brasil
ORCID: https://orcid.org/0000-0001-8453-017X
E-mail: ewerton.santos@ uncisal.edu.br
Ruth França Cizino da Trindade
ORCID: https://orcid.org/0000-0001-9932-6905
Universidade Federal de Alagoas, Brasil
E-mail: ruth.trindade@ @eenf.ufal.br
Juliane Maria Alves Siqueira Malta
ORCID: https://orcid.org/0000-0003-2581-5081
Hospital das Forças Armadas, Brasil
E-mail: maltajuliane@gmail.com
Marco Antônio Prado Nunes
ORCID: https://orcid.org/0000-0001-5244-5843
Universidade Federal de Sergipe, Brasil
E-mail: nunes.ma@ outlook.com
Francilene Amaral da Silva
ORCID: https://orcid.org/0000-0001-6729-2843
Universidade Federal de Sergipe, Brasil
E-mail: farmsilva @ hotmail.com

\section{Resumo}

Este estudo objetiva analisar a autocorrelação espacial das amputações de membros e as taxas de incidência de amputação entre os municípios de Alagoas. Estudo ecológico com dados do Departamento de Informática do SUS, as unidades de análise foram os municípios e a taxa de amputação. Foram incluídos casos de amputação de membros registrados entre 2007-2018. A taxa de incidência foi suavizada pelo método bayesiano local e empregou-se o índice de Moran para análise de dependência espacial no TerraView e mapas temáticos editados no QGis. Correlação de Spearman e modelo de regressão simples comparou as taxas com indicadores socioeconômicos regionais. Notificados 9345 registros de amputação no Estado. A estimativa bayesiana suavizou áreas e discriminou heterogeneidade com mais municípios de taxas elevadas e áreas de transição epidemiológica. A análise espacial de Moran exibiu quadriênio (2007-2010) valor do índice global 0.133385, (2011-2014) 0.215665, (2015-2018) 0.276478 prenunciando a evidência de autocorrelação espacial positiva. A análise de regressão univariada mostrou a taxa de incidência de amputação relacionada com indicadores que refletem situação de vida vulnerável da população, a taxa de desemprego (11,4\%), renda per capita $(8,5 \%)$ e taxa de analfabetismo masculino $(12,9 \%)$ percentuais de explicação. A distribuição espacial das amputações demonstrou um padrão de distribuição não homogêneo com aglomerações nas regiões do agreste e leste alagoano. Elas são as que mais sentem impacto das desigualdades na distribuição de renda, condições de educação e dificuldade de acesso aos serviços de saúde. O entendimento do crescimento geográfico das DCNT revelaas como um ponto crítico na saúde pública.

Palavras-chave: Amputação; Determinantes sociais da saúde; Diabetes Mellitus; Epidemiologia; Análise espacial; Doença crônica. 


\begin{abstract}
This study aims to analyze the spatial autocorrelation of limb amputations and amputation incidence rates among the municipalities of Alagoas. An ecological study with data from the SUS Department of Informatics, the units of analysis were the municipalities and the amputation rate. Limb amputation cases registered between 2007-2018 were included. The incidence rate was smoothed by the local Bayesian method and the Moran index was used for spatial dependence analysis in TerraView and thematic maps edited in QGis. Spearman correlation and simple regression model compared rates with regional socioeconomic indicators. 9345 amputation records notified in the state. The Bayesian estimate smoothed areas and discriminated heterogeneity with more municipalities with high rates and areas of epidemiological transition. Moran's spatial analysis exhibited quadrennial (2007-2010) global index value 0.133385, (2011-2014) 0.215665, (2015-2018) 0.276478 foreshadowing evidence of positive spatial autocorrelation. The univariate regression analysis showed the amputation incidence rate related to indicators that reflect the population's vulnerable life situation, the unemployment rate $(11.4 \%)$, per capita income $(8.5 \%)$ and male illiteracy rate $(12.9 \%)$ percentages of explanation. The spatial distribution of amputations showed a non-homogeneous distribution pattern with agglomerations in the agreste and eastern regions of Alagoas. They are the ones who feel the impact of inequalities in income distribution, education conditions and difficulty in accessing health services the most. Understanding the geographic growth of CNCDs reveals them as a critical point in public health.
\end{abstract}

Keyword: Amputation; Social determinants of health; Diabetes Mellitus; Epidemiology; Spatial analysis; Chronic disease.

\title{
Resumen
}

Este estudio tiene como objetivo analizar la autocorrelación espacial de las amputaciones de miembros y las tasas de incidencia de amputaciones entre los municipios de Alagoas. Estudio ecológico con datos del Departamento de Informática del SUS, las unidades de análisis fueron los municipios y la tasa de amputaciones. Se incluyeron los casos de amputación de miembros registrados entre 2007-2018. La tasa de incidencia se suavizó mediante el método bayesiano local y se utilizó el índice de Moran para el análisis de dependencia espacial en TerraView y los mapas temáticos editados en QGis. El modelo de correlación de Spearman y regresión simple comparó tasas con indicadores socioeconómicos regionales. 9345 registros de amputación notificados en el estado. La estimación bayesiana suavizó las áreas y discriminó la heterogeneidad con más municipios con altas tasas y áreas de transición epidemiológica. El análisis espacial de Moran exhibió un valor de índice global cuadrienal (2007-2010) 0.133385, (2011-2014) 0.215665, (2015-2018) 0.276478 presagiando evidencia de autocorrelación espacial positiva. El análisis de regresión univariado mostró la tasa de incidencia de amputación relacionada con indicadores que reflejan la situación de vida vulnerable de la población, la tasa de desempleo (11,4\%), el ingreso per cápita (8,5\%) y la tasa de analfabetismo masculino (12,9\%) porcentajes de explicación. La distribución espacial de las amputaciones mostró un patrón de distribución no homogéneo con aglomeraciones en las regiones agreste y oriental de Alagoas. Son los que más sienten el impacto de las desigualdades en la distribución del ingreso, las condiciones de educación y la dificultad para acceder a los servicios de salud. Comprender el crecimiento geográfico de las ECNT las revela como un punto crítico en la salud pública.

Palabra clave: Amputación; Los determinantes sociales de la salud; Diabetes Mellitus; Epidemiología; Análisis espacial; Enfermedad crónica.

\section{Introdução}

As Doenças Crônicas não Transmissíveis (DCNT) representam uma delicada questão na saúde mundial devido à sua morbimortalidade. Supõe-se que, anualmente, aproximadamente 36 milhões de óbitos no mundo são por DCNT, com destaque nas enfermidades circulatórias, respiratórias crônicas, diabetes mellitus e neoplasias malignas. Elas são descritas em quatro grupos de doenças: câncer, doenças cardiovasculares, doenças respiratórias crônicas e diabetes. As implicações sociais e econômicas do aumento destas doenças podem ser notadas, principalmente nas comunidades com maior sinal de fragilidade. Em 2011 no encontro das Nações Unidas, os chefes de Estado, admitiram publicamente que esses agravos à saúde são grande ameaça para as comunidades, enfatizando a relevância desta temática ser prioridade dentre os compromissos para o desenvolvimento, buscando soluções para reduzir a carga global das DCNT (Duncan et al., 2012; WHO, 2014).

Para compreender o aumento dessas doenças e a correlação com questões sociais e econômicas, faz-se necessário conhecer como as comunidades se transformaram no decorrer do tempo. A veemência dos acontecimentos como a transição demográfica e epidemiológica vem sendo relatada no Brasil e no mundo no decorrer dos últimos decênios, instigando relevantes questionamentos para gestores dos sistemas de saúde, no tocante a questões sociais do país e estruturais dos serviços de saúde. Nesse cenário destaca-se o papel dos Determinantes Sociais em Saúde (DSS) que são compostos pelos elementos que 
estão relacionados com os hábitos e às suas condições de vida e trabalho das pessoas, como também estão conectados com a macroestrutura econômica, social e cultural (Lucas, 2016).

A transição alimentar e nutricional relaciona-se diretamente com as mudanças socioeconômicas e consequentemente das transições demográfica e epidemiológica, gerando modificações no perfil de consumo alimentar, que levaram à diminuição da desnutrição e na elevação do excesso de peso e obesidade em idades distintas (Jaime \& Santos, 2014). Ela gerou mudança no modo de vida das pessoas que passaram a consumir mais alimentos industrializados, gordurosos, de preparo rápido (fast food), menos atividade física, e uma vida mais atribulada. Essa vida acelerada provocou avanços em várias áreas, mas também trouxe enfermidades que antes eram comuns nos mais velhos, como: diabetes mellitus (DM), hipertensão arterial sistêmica (HAS), neoplasias malignas, circulatórias e respiratórias crônicas e têm contribuído com a carga de doenças no Brasil, as quais se apresentam em várias faixas etárias, principalmente, nas mais jovens (Louzada et al., 2015; Canella et al., 2014).

Segundo Almeida et al. (2017) as doenças não transmissíveis são encarregadas por 66,0\% da carga de enfermidades no Brasil, com destaque para o Diabetes Mellitus por sua morbimortalidade. Em 2001, a incidência de amputações no Brasil era de 13,9 por 100.000 habitantes/ano, sendo que 80.900 amputações foram devido à DM, das quais 21.700 levaram o paciente à óbito. Entre 2011 e 2016, foram realizadas 102.056 cirurgias de amputação no SUS, das quais 70\% em pacientes com DM e a maioria (94\%) amputações mais baixas, que nesse mesmo período, no estado de Santa Catarina, houve 11.041 amputações de membros inferiores (Santos et al., 2018).

Os autores supracitados ainda destacam o último estudo Global Burden of Disease (GBD) que diz haver o aumento de anos de vida saudável perdidos. O estudo traz a preocupação com os anos de vida perdidos nas duas décadas seguintes, principalmente aqueles atrelados à DM e a problemas correlacionados, como por exemplo: retinopatia diabética, diabetes neuropatia, amputações e doença renal crônica. Conforme previsões do estudo do GBD, indivíduos com enfermidades crônicas vivem mais, porém com problemas como amputações devido a DM e diálise. Esse cenário chama a atenção para o fato de que a tendência é de mais anos de vida com deficiência; os quais podem ser onerosos para os pacientes, suas famílias e o sistema de saúde (Santos et al., 2018).

No Brasil, segundo Jesus-Silva et al. (2017), um estudo populacional com 5.539 pacientes submetidos a amputações maiores constatou uma incidência de 9,7 casos/100 mil habitantes na faixa de 30 a 89 anos e de 29,5 casos/100 mil habitantes de 55 a 74 anos, números que cresceram substancialmente quando pesquisados apenas os diabéticos (45,98 e 92,19 casos/100 mil habitantes, respectivamente). A média de idade para os pacientes pesquisados foi parecida com outras pesquisas populacionais, por volta de 65 anos. Essa mesma pesquisa brasileira atentou para a disposição equivalente das etiologias, com DM e doença arterial crônica periférica abordando a maioria dos pacientes $(90,7 \%)$, seguido do trauma $(5,6 \%)$, osteomielite $(1,7 \%)$, gangrena gasosa $(1,2 \%)$ e neoplasias $(0,8 \%)$.

Mesmo com o aumento da taxa de revascularização, a amputação de membro inferior ainda é uma intervenção predominante na prática clínica, o que pode estar relacionado com o aumento da expectativa de vida das pessoas que apresentam cada vez mais comorbidades. A taxa de mortalidade depois da amputação de membro inferior é considerada alta, podendo atingir 22\% após 30 dias, $44 \%$ após um ano e 77\% aos cinco anos (Rolim et al., 2015).

Para o grupo de pesquisa global dos membros inferiores, a amputação dos membros (AMI) é entendida como uma perda completa de qualquer parte do membro inferior. Nesse cenário, vale destacar que as taxas de amputação variam de maneira significativa no mundo e no Brasil. Aquelas estão entre 0,7 por mil na população do leste asiático e 31 por mil nos Estados Unidos das Américas (EUA). Comparando os indivíduos sadios (0,29\%), o diabetes apresenta de 10 a 30 vezes mais risco de AMI (2,8\%). Por causa do diabetes constata-se que um membro inferior é amputado no mundo a cada 30 segundos. Cerca de 60\% a 80\% das AMI não traumáticas estão sendo relatadas em indivíduos diabéticos e 85\% das AMI em diabéticos são precedidos por uma Úlcera no Pé Diabética (UPD) de cicatrização comprometida (Bal et al., 2019). 
As amputações de membros inferiores representam $85 \%$ do total e expressam um enorme impacto socioeconômico, além do comprometimento e/ou perda da capacidade laboral, da socialização e da qualidade de vida, apresentando complicações como: hematoma, infecções, necrose, contraturas, dor fantasma, reinternações, etc; caracterizando um relevante problema de saúde pública (Jesus-Silva et al., 2017).

Presume-se que mais de $80 \%$ das amputações sejam antecedidas por uma úlcera diabética no pé, sendo a DM a causa mais provável de amputação não traumática. Indivíduos com DM, apresentam-se com duas vezes mais chances de uma segunda amputação do que indivíduos sem diabetes. Além do mais, até $70 \%$ dos indivíduos amputados morrem dentro de cinco anos após a cirurgia e $44 \%$ morrem dentro de cinco anos após a cirurgia com uma outra úlcera no pé se não houver a amputação (Graz et al., 2018).

As complicações do pé diabético (CPD) são consequências relevantes do diabetes e corroboram para a maioria das causas de membros inferiores não traumáticos e amputações no mundo. Além do mais, pacientes com CPD são apontados por apresentarem maior taxa de recorrência de úlceras. Essa situação reporta-se a outro fator tão importante quanto: o custo. As despesas dos pacientes com complicações são ainda maiores do que as dos cânceres mais caros; além disso, os pacientes que tiveram AMI geralmente pioram as consequências apresentando baixa autoestima, vida útil reduzida e o ônus da assistência social (Lin et al., 2019).

Além das amputações de membros não traumáticas, as amputações de membros decorrentes de trauma também são vistas como uma pandemia da vida contemporânea e, ao que se percebe, tende a permanecer. Nesse cenário vê-se que a quantidade de vítimas aumenta, com destaque para àquelas na faixa etária de adultos jovens e economicamente operantes. Os danos seguem em iguais proporções com relevante impacto social quando resta sequela, como a amputação de um membro decorrente de danos sofridos. O avanço econômico das pessoas de nações em desenvolvimento, como o Brasil, e o fomento da produção da indústria automotiva vinculada a um quantitativo cada vez superior de veículos em trânsito acompanham-se de uma elevação assustadora de acidentes, que, regularmente, compreendem traumas graves dos membros (Senefonte et al., 2012).

O presente estudo se justifica pelo registro crescente de hospitalizações por trauma e complicações do DM no Brasil e em Alagoas, consequentemente, o aumento das amputações é o objeto deste estudo ecológico, pela necessidade de mais estudos semelhantes e a importância de obter evidências que ajudarão a compreender e dar visibilidade ao cenário epidemiológico das amputações em Alagoas. Partindo desse pressuposto, objetiva-se analisar a autocorrelação espacial das amputações de membros e as taxas de incidência de amputação entre os municípios no estado de Alagoas.

\section{Metodologia}

\section{Desenho de estudo e fonte dos dados}

Trata-se de estudo epidemiológico e ecológico. Segundo Freire e Pattussi (2018) os estudos ecológicos utilizam dados acerca de comunidades inteiras ou conglomerado de pessoas para verificar a regularidade da doença ou outra consequência entre distintos grupamentos, em um mesmo intervalo de tempo ou na mesma comunidade em momentos diferentes. Esse tipo de estudo além de ser rápido e de ter um gasto relativamente baixo tem descrito dessemelhanças em comunidades, as quais têm sido fundamentais para o planejamento das ações em saúde pública.

O Estado de Alagoas em 2002 (PDR, 2002) compreendia de 13 regiões de saúde (RS), e em concordância com o Plano Diretor de Regionalização de Alagoas - PDR (2011) foi reorganizado em 10 regiões de saúde e duas macrorregiões, as quais agrupam da $1^{\mathrm{a}}$ a $6^{\mathrm{a}} \mathrm{RS}$ a $1^{\mathrm{a}}$ macrorregião de saúde e da $7^{\mathrm{a}}$ a $10^{\mathrm{a}} \mathrm{RS}$ a $2^{\mathrm{a}}$ macrorregião de saúde, sendo os municípios de Maceió e Arapiraca referências para os serviços de saúde, respectivamente (figura 1). Vale destacar que a mesorregião do leste 
alagoano corresponde aos municípios que pertencem $1^{\mathrm{a}}$ macrorregião de saúde; a mesorregião do agreste responde pelas $7^{\mathrm{a}} \mathrm{e}$ $8^{\mathrm{a}}$, e o sertão pelas $9^{\mathrm{a}}$ e $10^{\mathrm{a}}$ regiões de saúde.

As amputações foram consideradas segundo município de residência no estado de Alagoas que está dividido em três mesorregiões geopolíticas - Leste, Agreste e Sertão, as quais apresentam diferentes características demográficas, econômicas e sociais. Ressalta-se que o estado tem 102 municípios que juntos somam 3.351.543 milhões de habitantes, população estimada para o ano de 2020, apresentando densidade demográfica de 112,33 hab. $/ \mathrm{km}^{2} \mathrm{em}$ uma área de $27.843 .295 \mathrm{~km}^{2}$. Em relação ao país, é o terceiro menor estado em território, respondendo por 0,3\% da área do Brasil. Faz fronteira com Pernambuco ao Norte, Sergipe ao Sul e Bahia ao Oeste (IBGE, 2019).

Os municípios do estado de Alagoas são as unidades de análise e seus respectivos registros de amputação de membros. Os 9.345 registros são de pacientes submetidos a amputação de membros superiores e/ou inferiores internados em hospitais conveniados com SUS no período de 2007 a 2018.

Figura 1 - Mesorregiões de Alagoas, 2021.

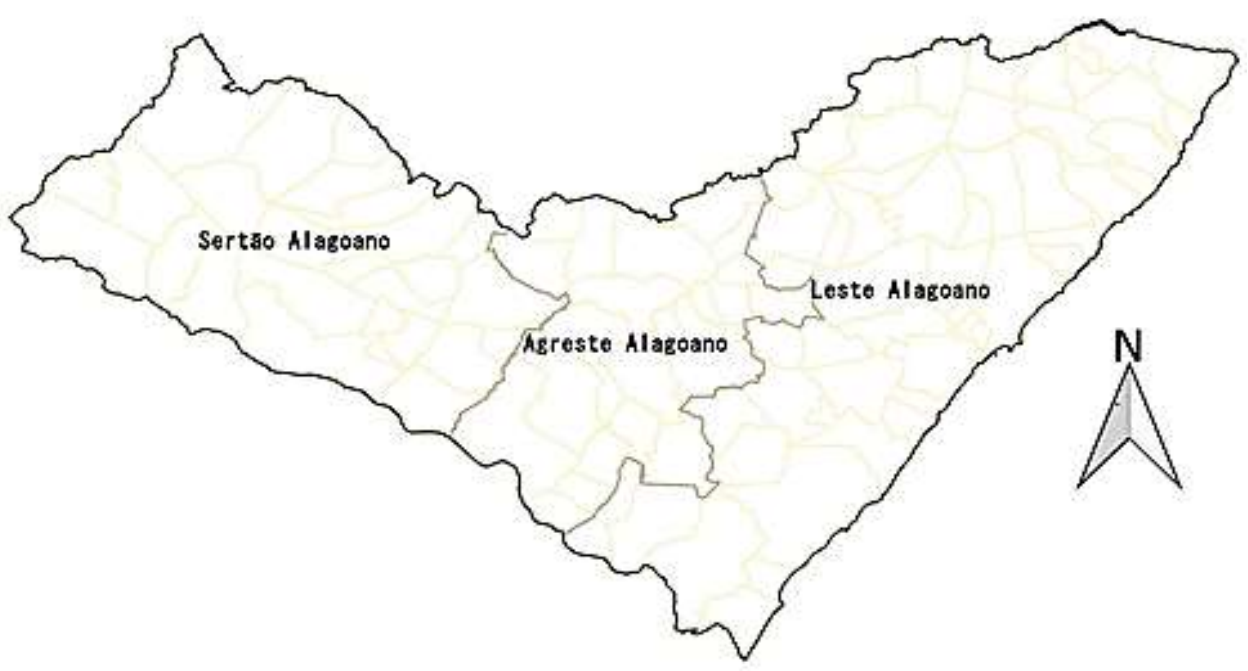

Fontes: Instituto Brasileiro de Geografia e Estatística (IBGE). https://www.ibge.gov.br/geociencias/organizacao-do-territorio/malhasterritoriais/15774-malhas.html?edicao=27415\&t=acesso-ao-produto.

QGIS (anteriormente conhecido como Quantum GIS) é um software livre com código-fonte aberto, multiplataforma de sistema de informação geográfica (SIG) que permite a visualização, edição e análise de dados georreferenciados. https://www.qgis.org/en/site/

O banco de dados de amputação de membros foi acessado por meio do Sistema de Informações Hospitalares do Sistema Único de Saúde (SIH/SUS), utilizando o programa TAB para Windows (TabWin) desenvolvido pelo Departamento de Informática do SUS (Datasus). Os dados populacionais foram provenientes do censo 2010 e estimativas para o ano de 2020 pelo Instituto Brasileiro de Geografia e Estatística (IBGE).

O estudo foi realizado com dados secundários já publicados em sistema de acesso público (DATASUS), respeitandose os princípios éticos constantes nas Resoluções do Conselho Nacional de Saúde n ${ }^{\circ} 466$ de 12 de dezembro de 2012, e $n^{\circ} 510$ de 7 de abril de 2016 dispensando sua apreciação por Comitê de Ética em Pesquisa.

\section{Variáveis}

Os municípios foram considerados no estudo por haver registros de amputação de membros (traumática ou não traumática). Os dados foram coletados em dezembro de 2019, considerando-se o limite temporal de janeiro de 2007 a dezembro de 2018. Entretanto, o ano de 2019 não foi considerado para este estudo porque ainda não se encontrava consolidado há época. 
Os indicadores socioeconômicos por retratarem parte do contexto de vulnerabilidade social e envolver expectativa de vida, economia e educação, como por exemplo: taxa de analfabetismo que corresponde ao percentual da população de 15 anos de idade ou mais que não sabem ler nem escrever no idioma conhecido; taxa de desemprego que corresponde ao percentual da população de 16 anos e mais ociosa em relação àqueles que trabalham; renda média domiciliar per capita que é o somatório dos rendimentos mensais dos residentes no domicílio, em reais, dividido pelo número de seus residentes (IBGE, 2010; DATASUS, 2010).

O índice de Desenvolvimento Humano Municipal (IDHM) composto de indicadores com três dimensões do desenvolvimento humano - longevidade, educação e renda per capita - agrupados por meio de média geométrica. A longevidade é mensurada a partir expectativa de vida ao nascer, calculada por método indireto a partir dos dados dos Censos Demográficos do IBGE. O IDHM retrata o número médio de anos que as pessoas viveriam contando do nascimento, mantidos os padrões de mortalidade observados no ano de referência. Já a educação é mensurada pela média de anos de educação de adultos, isto é, o número médio de anos de educação adquiridos no decorrer da vida por pessoas a partir de 25 anos de idade; e índice de Gini que é a diferença entre os rendimentos médios mensais das pessoas de 15 anos ou mais de idade com rendimento, permitindo calcular a desigualdade de distribuição de renda (IBGE, 2010; DATASUS, 2010).

\section{Análise dos Dados}

Foi construída a série histórica de doze anos - 2007 a 2018 das internações que tiveram como desfecho a amputação de membros. O banco de dados foi compilado no Software Microsoft Office Excel, versão 2013.

A análise espacial foi realizada a partir da base cartográfica do Estado de Alagoas, disponível no banco de dados do IBGE (malhas territoriais). Utilizou-se o Terra Datum modelo SIRGAS 2000 e a projeção cartográfica correspondente ao sistema Mercator Transversal Universal. Os dados georreferenciados foram analisados nos softwares TerraView (Instituto Nacional de Pesquisas Espaciais - INPE, São Paulo/BR 4.2.2) e Quantum GIS (Open Source Geospatial Foundation, OSGeo, CHI, EUA, Versão 2.18.2).

Os mapas de análise espacial foram construídos a partir das taxas de incidência, com a média das amputações registradas a cada quatro anos por município. As taxas de incidência foram suavizadas pelo método bayesiano empírico local para corrigir flutuações aleatórias em populações ou números de ocorrência pequenos por meio de uma reestimativa que considera existir autocorrelação entre taxas de áreas vizinhas. Para isso, obteve-se uma média ponderada entre o valor medido e a taxa média da vizinhança, com pesos inversamente proporcionais à população de cada área.

Para estimar a variabilidade do espaço na análise dos dados, uma matriz de proximidade foi construída, na qual os municípios adjacentes receberam o valor 1 (um) e os que não o fizeram geometrias de fronteira adjacentes foram categorizadas com o valor 0 (zero). O índice de Moran Global por ser uma medida que fornece a significância estatística foi utilizado para identificar autocorrelações espaciais entre as áreas consideradas no estudo por meio de valores que variam de -1 a 1 , indicam aglomerados de regiões intermediárias de articulação urbana (RAU) com riscos semelhantes para ocorrência da amputação. Os índices positivos retratam a existência de autocorrelação espacial entre municípios vizinhos, que compartilham a mesma fronteira; os valores negativos indicam ausência de similaridade entre os mesmos e os índices próximos a zero significam ausência de correlação espacial. Foi adotado um nível de significância de 95\%.

O mapa de Moran mostra RAU críticas e transicionais estatisticamente significativas, indicando as categorias dos clusters (um conjunto conexo de regiões do mapa onde o risco de ocorrência dos eventos é elevado) e suas relações com os vizinhos. Esta análise verifica a existência de dependência espacial e padrões de risco de amputação pela criação de diagramas de espalhamento e dos quadrantes espaciais Q1 (alta + / alta +) RAU mais crítica, Q2 (baixo - / baixo -) RAU menos crítica, que apresentam municípios com valores equivalentes aos dos seus vizinhos, Q3 (alto + / baixo -) e Q4 (baixo - / alto +) RAU 
dissimilares. As duas primeiras classes representam áreas de concordância com agregados de associação espacial positiva e as duas últimas mostram áreas dissimilares com agregados de associação espacial.

Foi verificada a correlação entre a taxa de incidência de amputação de membro e os seguintes indicadores socioeconômicos: IDHM, índice de Gini, renda per capita, taxa de analfabetismo, taxa de analfabetismo feminino, taxa de analfabetismo masculino e taxa de desemprego. Utilizou-se a seguinte interpretação da direção e força da correlação conforme o coeficiente de Spearman (Callegari-Jacques, 2009): -1 (perfeita e negativa); - (>0 e/ou $\leq 0,3$ ) (fraca e negativa); - (>0,3 e/ou $\leq 0,6)$ (moderada e negativa); - $(>0,6 \mathrm{e} / \mathrm{ou} \leq 0,9)$ (forte e negativa); - $(>0,9 \mathrm{e} / \mathrm{ou}<1,0)$ (muito forte e negativa); 0 (nula); $>0$ e/ou $\leq 0,3$ (fraca e positiva); $>0,3 \mathrm{e} / \mathrm{ou} \leq 0,6$ (moderada e positiva); $>0,6 \mathrm{e} / \mathrm{ou} \leq 0,9$ (forte e positiva); $>0,9 \mathrm{e} / \mathrm{ou}<1,0$ (muito forte e positiva) e 1 (perfeita e positiva). Para estimar o modelo de regressão de acordo com os indicadores socioeconômicos dos municípios foi realizada a análise de regressão linear.

\section{Resultados}

Do total de amputações $(\mathrm{N}=9.345)$ somente no terceiro quadriênio (2015-2018) foram registradas $5.210(55,75 \%)$. A taxa de incidência média de amputação em Alagoas no período estudado foi de 145,11 amputações por 100 mil habitantes. Foram identificados 18 (17,65\%) municípios no primeiro quadriênio (A) situados na região do sertão, no agreste e leste alagoano com taxas de incidência críticas, mais elevadas que a média estadual, que variaram de 82,0 a 219,1 amputações por 100 mil habitantes. O segundo quadriênio (B) apresenta 19 (18,63\%) municípios situados no agreste e leste alagoano com taxas de incidência críticas, mais elevadas que a média estadual, que variaram de 77,0 a 229,1 amputações por 100 mil habitantes.

No terceiro quadriênio (C) observa-se que os municípios se concentram mais no leste alagoano, com $21(20,60 \%)$ deles apresentando taxas de incidência críticas, mais elevadas que a média estadual que variaram de 163,0 a 204,0 amputações por 100 mil habitantes. No entanto, vê-se outros 20 (19,60\%) municípios com taxas de incidência ainda mais críticas, com elevação da média em torno de $29 \%$ que a média do estado de Alagoas variando de 204,0 a 572,1 amputações por 100 mil habitantes.

Os municípios de Cacimbinhas, Pilar, Rio Largo, Coqueiro Seco, Matriz de Camaragibe chamam a atenção porque estão com taxas de incidência críticas, mais elevadas que a média do Estado nos três quadriênios, com valores ainda maiores no terceiro quadriênio. A figura 2 retrata a distribuição espacial da taxa de incidência média para os três quadriênios, na qual é perceptível o surgimento de clusters nos municípios comuns aos três. 
Figura 2 - Taxas médias de incidência das amputações por município de residência. Alagoas. A) 2007-2010 B) 2011-2014 C) 2015-2018.

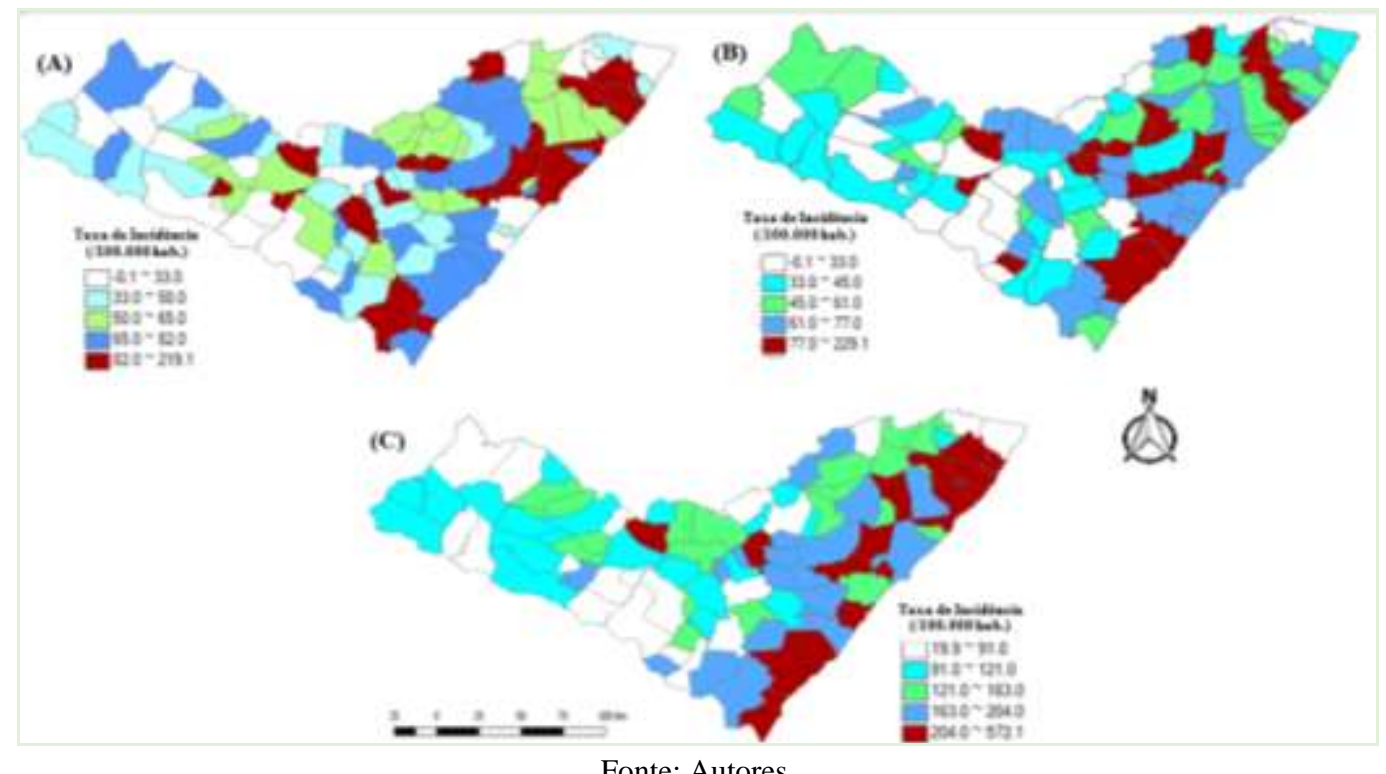

Fonte: Autores.

O ajuste da taxa de incidência pela análise estimativa bayesiana empírica local amenizou as áreas e revelou uma heterogeneidade com maior número de municípios revelando taxas elevadas: Jacaré dos Homens, Olho D’Água Grande, Belém, Mar Vermelho, Piaçabuçu, Marechal Deodoro, Paripueira, Flexeiras todos no primeiro quadriênio (A); Mata Grande, Minador do Negrão, Major Isidoro, Feliz Deserto, Novo Lino, Porto de Pedras, São Miguel dos Milagres no segundo quadriênio (B); Santana do Mundaú, Murici, Penedo no terceiro quadriênio (C). Ressalta-se que Mar Vermelho e Marechal Deodoro mantêm seus coeficientes de detecção elevados nos três quadriênios.

Esse cenário se destaca na região leste, mas também no agreste e sertão timidamente segundo mapas temáticos da Figura 3. Nas estimativas brutas, não atenuadas, alguns municípios apresentam incidências díspares de seus vizinhos, o que, conforme o primeiro mapa, dificulta a visualização de uma tendência de gradiente de incidência em larga escala devido a flutuações aleatórias provocadas por efeitos populacionais reduzidos. 
Figura 3 - Taxas médias de incidência das amputações corrigidas pelo modelo empírico Bayesiano local, Alagoas. A) 20072010 B) 2011-2014 C) 2015-2018.

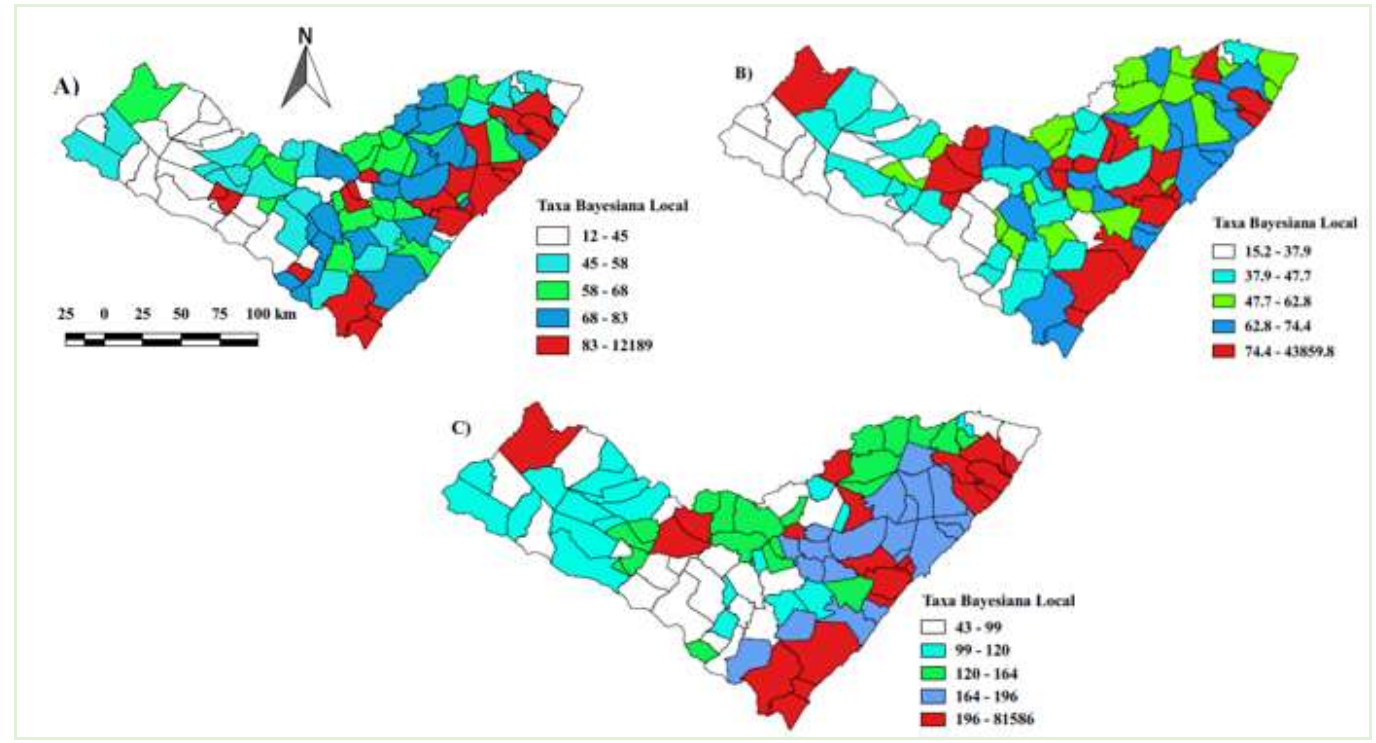

Fonte: Autores.

A análise espacial de Moran exibiu no quadriênio 2007-2010 valor do índice global de 0,133385 ( $p$-valor = 0,01), no quadriênio de 2011-2014 índice global de 0,215665 ( $p$-valor = 0,01) e no quadriênio 2015-2018 índice global de 0,276478 ( $p$ valor $=0,02)$ prenunciando a evidência de autocorrelação espacial positiva entre os municípios. Dessa forma, os municípios vizinhos inclinam-se a ser semelhantes entre si no tocante à variável taxa de incidência.

Ao analisar o índice global de associação espacial os clusters foram observados predominantes na região leste alagoana (Figura 4) que se ampliaram no segundo quadriênio com 31 municípios $(30,4 \%)$. No terceiro quadriênio observa-se também o aumento dos clusters com 35 (34,3\%) municípios predominantes no leste alagoano.

Figura 4 - Box Map das taxas médias de incidência das amputações por município de residência, Alagoas. A) 2007-2010 B) 2011-2014 C) 2015-2018.

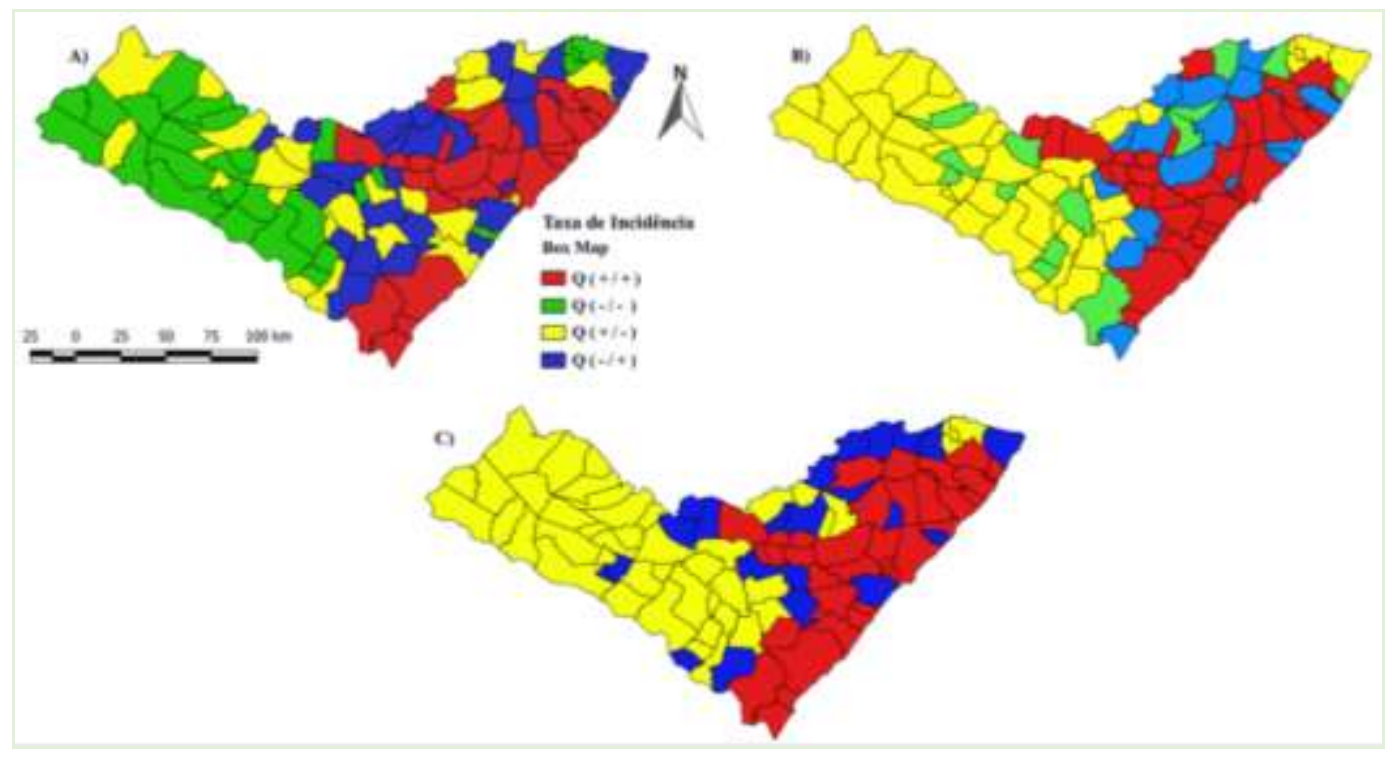

Fonte: Autores. 
As informações do Moran Map identificam conglomerados com significância estatística classificados conforme sua posição no diagrama de espalhamento (Figura 5). No terceiro quadriênio observa-se que há municípios que, mesmo nas regiões intermediárias de articulação urbana (RAU) (Q3 e Q4 no Moran Map), saíram de Q4 para Q3: Piranhas, Poço das Trincheiras, Jacaré dos Homens e Paulo Jacinto. Entretanto, Porto de Pedras, Pindoba e Maribondo tiveram movimentação inversa de Q3 para Q4. Foram visualizadas áreas em fase de transição com alguns agrupamentos no terceiro quadriênio, mas há muitos desagrupados por conterem taxas diferentes dos circunvizinhos, representadas por altos e baixos valores de incidência de amputações.

Figura 5 - Moran Map das taxas médias de incidência das amputações por município de residência, Alagoas. A) 2007-2010 B) 2011-2014 C) 2015-2018.

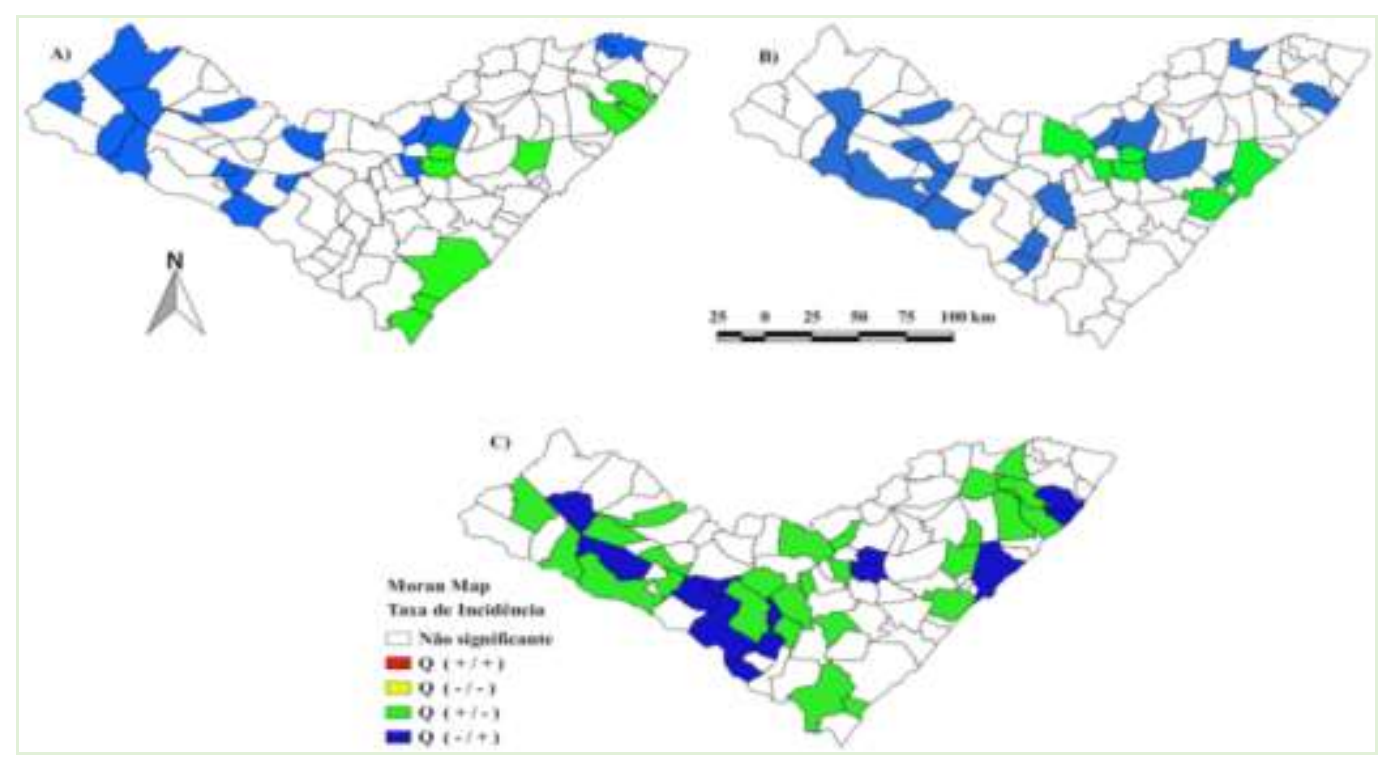

Nota: Q3 equivale a Q (+ / -) e Q4 equivale a Q (- / +). Fonte: Autores.

No tocante aos indicadores socioeconômicos foi encontrada correlação moderada e positiva entre IDHM, renda per capita, taxa de desemprego e a taxa de incidência de amputação. Entretanto, a taxa de analfabetismo masculino apresenta-se com correlação moderada e negativa, sinalizando que a taxa de incidência das amputações tende a aumentar à medida que a taxa de analfabetismo masculino diminui. Foram evidenciadas as variáveis socioeconômicas com correlação estatisticamente significativa $(p$-valor $\leq 0,05)$ segundo Tabela 1 e Figura 6.

Tabela 1 - Coeficiente de correlação de Spearman entre a taxa de incidência de amputação de membro e indicadores socioeconômicos de Alagoas 2007-2018.

\begin{tabular}{lcc}
\hline \multicolumn{1}{c}{ Indicadores socioeconômicos } & $\mathbf{R}$ & p-valor \\
\hline IDHM & $0.371^{* *}$ & $<0.001$ \\
Índice de Gini & $-0.352^{*}$ & $<0.001$ \\
Renda per capita & $0.468^{* *}$ & $<0.001$ \\
Taxa de analfabetismo & $-0.392^{*}$ & $<0.001$ \\
Taxa de analfabetismo feminino & $-0.281^{*}$ & 0.004 \\
Taxa de analfabetismo masculino & $-0.446^{*}$ & $<0.001$ \\
Taxa de desemprego & $0.485^{* *}$ & $<0.001$ \\
\hline
\end{tabular}

Notas: *IBGE referentes ao Censo Demográfico 2010. ** Datasus de 2007 a 2018. Fonte: IBGE (2010). 
A análise de regressão univariada mostrou que a taxa de incidência de amputações de membros por 100.000 habitantes esteve relacionada com indicadores que refletem situação de vida vulnerável da população durante período do estudo, a taxa de desemprego $(p=0,001)$, renda per capita $(p=0,001)$ e taxa de analfabetismo masculino $(p=0,001)$ (Tabela 2). No entanto, os coeficientes de determinação indicam percentuais de explicação $11,4 \%, 8,5 \%$ e $12,9 \%$, respectivamente, da variabilidade da taxa de incidência diante dessa variável independente.

Tabela 2. Regressão linear simples global entre taxa de incidência das amputações de membros e indicadores socioeconômicos. Alagoas, Brasil, 2007-2018.

\begin{tabular}{cccc}
\hline Indicador Socioeconômico & Modelo de regressão & $\mathbf{R}^{2}$ & $\boldsymbol{p}$-valor \\
\hline IDHM & $\mathrm{y}=0,0003 \mathrm{x}+0,53$ & 0,1082 & 0,001 \\
Taxa de desemprego & $\mathrm{y}=0,0377 \mathrm{x}+5,78$ & 0,1145 & 0,001 \\
Renda per capita & $\mathrm{y}=0,5647 \mathrm{x}+195,66$ & 0,0859 & 0,001 \\
Taxa de analfabetismo masculino & $\mathrm{y}=0,0679 \mathrm{x}+40,17$ & 0,1290 & 0,001 \\
\hline
\end{tabular}

Fonte: Autores. 
Figura 6. Gráficos de dispersão com a variável dependente (taxa de incidência de amputação) e as variáveis socioeconômicas dos municípios de Alagoas, Brasil.
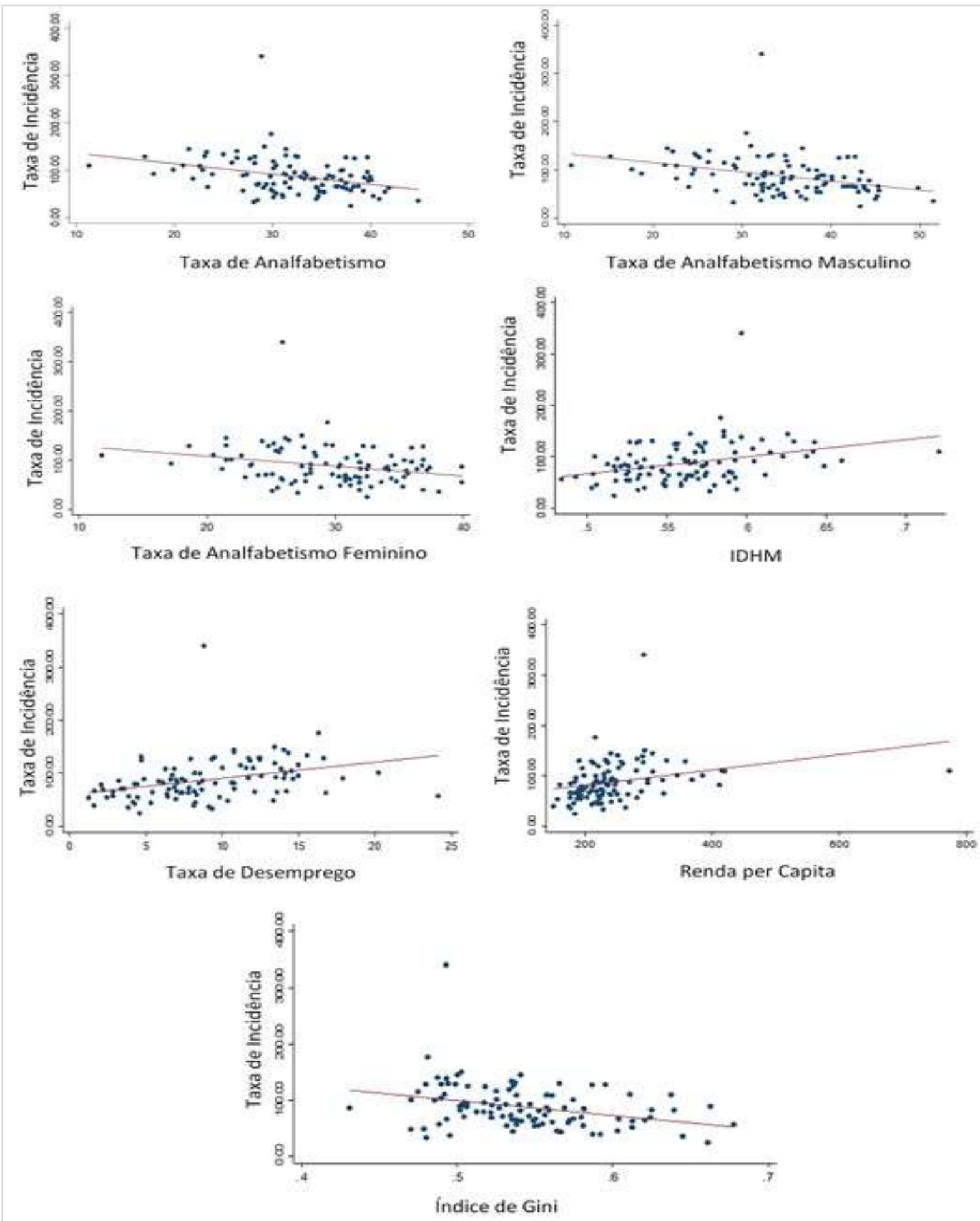

Fonte: Autores.

\section{Discussão}

A análise da organização espacial proporcionou a visualização das áreas dos municípios com maior aglomeração de amputação. As técnicas de geoprocessamento indicaram a presença maior das taxas de incidência de amputação nas regiões agreste e leste, principalmente no leste alagoano. Certamente isso se deu porque a dispersão dos procedimentos dentro do 
estado de Alagoas a partir de 2013 foi um fenômeno relevante pois surgiram novos estabelecimentos hospitalares e redistribuição das autorizações de internação hospitalar (AIH); como também se observou o aumento na relação número de amputações por habitante e a manutenção do maior percentual de procedimentos realizados em membros inferiores (Peixoto et al., 2017).

Esta grande área geográfica de aglomeração das amputações está nas proximidades de praias, regiões turísticas e da capital do estado. Deve-se ressaltar que, apesar de o mapa indicar um padrão espacial distinto, retratado pela variação de cores, observa-se municípios com taxa de incidência de amputação muito maiores do que a do estado.

Para analisar padrões espaciais de maneira mais minuciosa calculou-se o índice de associação espacial às regiões, proporcionando o reconhecimento de aglomerados (clusters) no território, mostrando as regiões no estado que indicam as maiores semelhanças de disposição das amputações. Vale ressaltar que as regiões que já demonstravam esse tipo de aglomeração mantiveram-se assim no decorrer dos quadriênios.

Dentre os quatro municípios (Jacaré dos Homens, Paulo Jacinto, Piranhas e Poço das Trincheiras) que mudaram de quadrante (Q4 para Q3) na análise espacial do Moran Map, mesmo se mantendo em uma região intermediária de articulação urbana dissimilar, observa-se um aumento na taxa de incidência das amputações, tornando-a maior do que dos municípios vizinhos. Apesar de pertencerem ao sertão alagoano, com um percentual de cobertura de estratégia de saúde da família (ESF) em média de 97,30\% o que não justificaria essa elevação (Ministério da Saúde, 2021). Questões culturais e/ou comportamentais possivelmente possam elucidar tais resultados visto que os habitantes do sertão alagoano tendem a possuir hábitos inadequados como sedentarismo, dieta hipercalórica, hiper sódica.

Dentre os três municípios (Maribondo, Pindoba e Porto de Pedras) que mudaram de quadrante (Q3 para Q4) na análise espacial do Moran Map, mesmo se mantendo em uma região intermediária de articulação urbana dissimilar, nota-se que eles tiveram uma redução na taxa de incidência das amputações, tornando-a menor do que dos municípios vizinhos. Observa-se que Maribondo pertence ao agreste, enquanto Pindoba e Porto de Pedras são do leste alagoano e possuem um percentual de 100\% de cobertura de ESF (Ministério da Saúde, 2021). Ressalta-se ainda a importância do impacto das questões culturais e/ou comportamentais nos resultados de cada região, visto as diferenças encontradas entre as três mesorregiões do estado de Alagoas.

Dentre as variáveis socioeconômicas elencadas os IDHM encontram-se abaixo de 0,7 com atenção para o IDHM Educação que sem mantém em média abaixo de 0,5 ; já o IDHM referente somente à longevidade nos três quadriênios se encontra em média acima de 0,7. Isso indica que a população está vivendo mais anos e precisará de maior infraestrutura (saúde, educação, emprego, segurança, alimentação, lazer, moradia, mobilidade urbana, etc) à medida que os anos aumentarem. Assim, outros problemas de saúde também poderão ser potencializados como as DCNT, as quais se não forem bem gerenciadas contribuirão para o aumento das amputações (PNUD, 2010).

Os coeficientes de determinação indicam percentuais de explicação das amputações, com destaque para taxa de analfabetismo masculino com 12,9\%, taxa de desemprego com 11,4\%, IDHM com 10,8\% e renda per capita com 8,5\%. No entanto, somente a taxa de analfabetismo masculino tem uma correlação negativa e moderada com a taxa de amputação de membros, o que significa dizer que quanto menos instrução se tem, mais amputações tendem a ocorrer. Henry et al. (2013) e Peek (2011) relatam que em um estudo de coorte retrospectivo com 364 pacientes submetidos à amputação não traumática de extremidades inferiores e em uma revisão sistemática mostraram que a maioria era do sexo masculino e com diagnóstico de diabetes mellitus têm maior chance de serem submetidos ao procedimento de amputação.

Humphries et al. (2016) colocam também que as consequências do cuidado também podem ser afetadas pela capacidade do usuário (ou família) executar o cuidado com a ferida, assim como estimular a baixa e/ou ausência de adesão aos medicamentos, e existência de transportes precários até o serviço de saúde ou barreiras geográficas. 
Houve um aumento no número de amputações entre os anos de 2014 a 2016 e uma redução em 2018 no estado. Este cenário é abordado por Peixoto et al. (2017) quando citam que o quantitativo de usuários com amputações aumenta de maneira alarmante, tendo como principais situações de risco às doenças vasculares, diabetes mellitus, fumo, hipertensão arterial sistêmica e trauma. Esta situação fica mais alarmante e preocupante quando se considera as sequelas socioeconômicas decorrentes das lesões sofridas, oriundas da perda da capacidade laborativa, da socialização e consequentemente da qualidade de vida, relacionada com a morbidade, incapacidade e mortalidade.

A amputação de membro inferior é um relevante indicador de qualidade da assistência prestada a usuários com diabetes mellitus, assim como as demais DCNT e por isso muitos países europeus, do Oriente Médio e africanos, além da Organização Mundial de Saúde e da Federação Internacional de Diabetes, têm determinado metas e objetivos para diminuir as taxas de amputações em até 50\%, e assim aprimorar os parâmetros de qualidade da assistência (Rodrigues, 2015).

O SUS oferece serviços públicos de saúde que permite acesso gratuito e universal a toda a população. Sua assistência é descentralizada e compete aos municípios a responsabilidade pela gestão da atenção primária desses serviços. O SUS responde por cerca de $77 \%$ das consultas ambulatoriais no país e cerca de $65,7 \%$ das internações hospitalares são custeadas por ele (Toscano et al., 2018).

O Ministério da Saúde (2016) reitera que a atenção primária é o nível ideal para a supervisão contínua da pessoa com DCNT: a atenção primária é o ambiente de assistência mais próximo da população e é encarregada pela assistência longitudinal e integral. Logo, a atenção primária é capaz de solucionar mais de $80 \%$ dos problemas de saúde da população, por isso deveria ser a porta de entrada preferencial do usuário no SUS. A equipe da atenção primária é responsável juntamente com os gestores pela avaliação da sua demanda.

Diante disso deve-se refletir sobre a relevância das atribuições dos serviços de saúde, como também da segurança de trânsito e do trabalho, nas intervenções educativas dos usuários e dos profissionais das várias áreas de atuação na atenção primária e na rede de atenção à saúde (RAS), no que se refere à prevenção das doenças vasculares, dos acidentes no trânsito e no trabalho, uma vez que estão entre as principais causas de amputações em Alagoas (Peixoto et al., 2017).

A Sociedade Brasileira de Diabetes (2019) afirma que o diabetes mellitus tem dado sua contribuição com o aumento de sua prevalência vinculado a vários fatores como a célere urbanização, transição epidemiológica, nutricional, aumento do sedentarismo, excesso de peso, envelhecimento populacional e, também, à maior sobrevida dos usuários com diabetes mellitus. Segundo o IBGE (2019) a expectativa de vida ao nascer para os homens era de 73,1 anos, para as mulheres 80,1 anos no Brasil e para Alagoas de 72,7 anos.

Esse estudo apresenta limitações por utilizar dados secundários, os quais podem receber influência da capacidade operativa do sistema de vigilância e da qualidade e rigor dos registros, principalmente no Brasil, onde o diagnóstico de diabetes mellitus pode ser minimizado na alta hospitalar. Além de pacientes com doença coronariana, cardíaca, vascular periférica, renal crônica, HAS, cerebrovascular, insuficiência cardíaca ou fibrilação arterial podem mascarar um diagnóstico complementar de diabetes mellitus. Outro fator relevante é que o banco de dados não permitiu precisar o quantitativo real de pessoas, uma vez que nele estão os registros dos procedimentos/amputações realizadas que podem ser da mesma pessoa em períodos diferentes de tempo partindo do princípio de que a reamputação é uma realidade muita próxima do paciente com DCNT principalmente com diabetes mellitus.

\section{Considerações Finais}

A distribuição espacial das amputações no estado demonstrou um padrão de distribuição não homogêneo, onde há regiões com taxa de incidência de amputação bem maior que em outras, nos períodos de estudo, com aglomerações nas regiões 
do agreste e leste alagoano com destaque para o leste. Essas regiões são as que mais retratam o cenário das desigualdades na distribuição de renda, condições de educação e dificuldade de acesso aos serviços de saúde.

É necessário a reflexão acerca das necessidades de melhoria das intervenções de cuidado para prevenir e/ou diminuir as lesões de membros inferiores, bem como ampliar a imprescindibilidade de elevação da autoconsciência e do compromisso pessoal entre homens. Partindo do pressuposto de que as diferenças no risco e proteções padrão de atitudes são um indicativo de vulnerabilidade masculina, havendo a necessidade de delinear e implementar estratégias de educação em saúde para ele.

O entendimento do crescimento geográfico das DCNT, quer pela elevação da expectativa de vida da população, quer pela transição alimentar e epidemiológica ou pela mudança nos hábitos socioculturais, e a ausência de controle dos fatores de risco, das questões sociais e financeiras implicadas caracterizam o tratamento das DCNT um desafio para toda a sociedade. O envolvimento coletivo com equipes multidisciplinares, dando ênfase a questões pessoais e atentas às consequências sociais, levará a todos a um esforço único, transpondo obstáculos individuais e vendo as DCNT como um ponto crítico na saúde pública.

Assim, vê-se a necessidade de maior fomento aos programas específicos para o público em questão com Estratégias de Saúde da Família capacitadas para que possam melhor garantir a aderência do portador de DCNT ao tratamento. A atenção primária deveria ser a primeira porta de entrada desse usuário no SUS, haja vista que ela pode proporcionar melhor disposição e andamento para os serviços da rede de atenção à saúde. Estas intervenções tendem a contribuir positivamente para a saúde física e mental dos portadores de DCNT e tendem também a diminuir os valores vultosos pagos pelo SUS.

Para estudos futuros recomenda-se a incorporação ou desenvolvimento de sistemas de informação que sejam capazes de armazenar dados individuais de pacientes e procedimentos realizados de forma que possam diminuir a duplicidade de informações, assegurar o espaço temporal informado, assim como complicações, doenças associadas e outros indicadores relevantes para esses estudos. Sugere-se também que outras pesquisas possam ser desenvolvidas analisando os processos de alimentação desses sistemas de informação, fomento de capacitações técnica dos profissionais responsáveis por essas atividades, visando a otimização, a veracidade, objetividade e coerência dos dados.

\section{Referências}

Almeida, V. C. D., Araújo, S. T., Negreiros, F. D. S., Aguiar, M. I. F., Moreira, T. R., Crispim, A. P. P. (2017). Micro and macro vascular complications in people with type 2 diabetes mellitus in outpatient care. Rev Rene. 18(6):787-93. http://DOI:10.15253/2175-6783.2017000600012.

Bal, B. S., Khanna, G., Bhardwaj, A., \& Singh, K. (2019). Evaluation of risk factors for lower extremity amputation in diabetic foot ulcer: a hospital based observational study in Northern India. Int J Res Med Sci. 7(4), 1190-1195. http://dx.doi.org/10.18203/2320-6012.ijrms20191323.

Callegari-Jacques, S. M. (2009). Bioestatística: princípios e aplicações. Traducao. Artmed Editora.

Canella, D. S., Levy, R. B., Martins, A. P. B., Claro, R. M., Moubarac, J-C, Baraldi, L. G., \& Monteiro, C. A. (2014). Ultra-processed food products and obesity in Brazilian households (2008-2009). PLoS One, 9(3), p. e92752. https://doi.org/10.1371/journal.pone.0092752.

DATASUS (2010). Departamento de Informática do SUS (DATASUS). http://datasus.saude.gov.br/.

Duncan, Bruce Bartholow, Chor, D., Aquino, E. M. L., Bensenor, I. M., Mill, J. G., Schimidt, M. I., \& Barreto, S. M. (2012). Chronic Non-Communicable Diseases in Brazil: priorities for disease management and research. Revista de Saúde Pública. 46(suppl), pp. 126-134. <https://doi.org/10.1590/S0034$89102012000700017>$

Freire, M. C. M., \& Pattussi, M. P. (2018). Tipos de estudos. In: C. Estrela (Org.). Metodologia Científica. Ciência, ensino e pesquisa. (3a. ed.) 109-127. Porto Alegre: Artes Médicas.

Graz, H., D'Souza, V. K., Alderson, D., \& Graz, M. (2018). Diabetes-related amputations create considerable public health burden in the UK. Diabetes research and clinical practice, 135, 158-165. https://doi.org/10.1016/j.diabres.2017.10.030.

Henry, A. J., Hevelone, N. D., Hawkins, A. T., Watkins, M. T., Belkin, M., \& Nguyen, L. L. (2013). Factors predicting resource utilization and survival after major amputation. Journal of vascular surgery, 57(3), 784-790. https://doi.org/10.1016/j.jvs.2012.09.035.

Humphries, M. D., Brunson, A., Li, C. S., Melnikow, J., \& Romano, P. S. (2016). Amputation trends for patients with lower extremity ulcers due to diabetes and peripheral artery disease using statewide data. Journal of vascular surgery, 64(6), 1747-1755.e3. https://doi.org/10.1016/j.jvs.2016.06.096. 
IBGE (2010). Instituto Brasileiro de Geografia e Estatística (IBGE). Censo Demográfico. https://www.ibge.gov.br/estatisticas/sociais/educacao/9662-censodemografico-2010.html?t=destaques.

IBGE (2019). Instituto Brasileiro de Geografia e Estatística (IBGE). Em 2019, expectativa de vida era de 76,6 anos. https://agenciadenoticias.ibge.gov.br/agencia-sala-de-imprensa/2013-agencia-de-noticias/releases/29502-em-2019-expectativa-de-vida-era-de-76-6anos\#: :text=A\%20expectativa\%20de\%20vida\%20dos,2018\%20(12\%2C4).

Jaime, P. C., \& Santos, L. M. P. (2014). Nutritional transition and the organization of care in food and nutrition in Primary Health Care. Divulgação em Saúde para Debate, (51), p. 72-85.

Jesus-Silva, S. G., Oliveira, J. P., Brianezi, M. H. C., Silva, M. A. M., Krupa, A. E., \& Cardoso, R. S. (2017). Analysis of risk factors related to minor and major lower limb amputations at a tertiary hospital. J Vasc Bras. 16(1), 16-22. https://doi.org/10.1590/1677-5449.008916.

Lin, C., Armstrong, D. G., Lin, C., Liu, P., Hung, S., Lee, S., \& Huang, Y. (2019). Nationwide trends in the epidemiology of diabetic foot complications and lower-extremity amputation over an 8-year period. BMJ Open Diabetes Research and Care. 7, e000795. https://doi: 10.1136/bmjdrc-2019-000795.

Louzada, M. L. C., Martins, A. P. B., Canella, D. S., Baraldi, L. G., Levy, R. B. Claro, R.M., \& Monteiro, C. A. (2015). Ultra-processed foods and the nutritional dietary profile in Brazil. Revista de Saúde Pública, 49(38), 1-11. https://doi.org/10.1590/S0034-8910.2015049006132.

Lucas, E. T. O. D. (2016). Distribuição espaço-temporal da prevalência de pé diabético e amputações por diabetes no Brasil antes e após a Lei nº 11.347/06. Dissertação (mestrado) - Universidade Federal do Rio Grande do Norte. Centro de Ciências da Saúde. Programa de Pós-Graduação em Saúde Coletiva, Natal.

Ministério da Saúde (2016). Secretaria de Atenção à Saúde. Departamento de Atenção Básica. Manual do pé diabético: estratégias para o cuidado da pessoa com doença crônica /Ministério da Saúde. http://189.28.128.100/dab/docs/portaldab/publicacoes/manual_do_pe_diabetico.pdf.

Ministério da Saúde (2021). Secretaria de Atenção Primária à Saúde (SAPS). PAINÉIS DE INDICADORES - Atenção Primária à Saúde. https://sisaps.saude.gov.br/painelsaps/saude-familia.

Peek, M. E. (2011). Gender Differences in Diabetes-related Lower Extremity Amputations. Clinical Orthopaedics and Related Research, 469(7), 19511955. https://doi.org/10.1007/s11999-010-1735-4.

Peixoto, A. M., Zimpel, S. A., Oliveira, A. C. A., Monteiro, R. L. S., \& Carneiro, T. K. G. (2017). Prevalence of upper and lower limb amputations of SUS patients in the state of Alagoas between 2008 and 2015. Fisioter Pesqui. 24(4), 378-384. https://doi:10.1590/1809-2950/17029524042017.

Plano Diretor de Regionalização da Saúde de Alagoas (2009). Plano Diretor De Regionalização Da Saúde Do Estado De Alagoas - PDR/AL. http://cidadao.saude.al.gov.br/transparencia/observatorios/observatorio-das-despesas-publicas-da-saude/monitoramento-da-saude/plano-diretor-deregionalizacao-pdr/.

Plano Diretor de Regionalização da Saúde de Alagoas (2011). Plano Diretor De Regionalização Da Saúde Do Estado De Alagoas - PDR/AL - Revisão no Ano 2011. https://www.saude.al.gov.br/wp-content/uploads/2020/06/pdr_2011.pdf.

PNUD (2010). Programa das Nações Unidas para o Desenvolvimento (PNUD). Índice de Desenvolvimento Humano. https://www.br.undp.org/content/brazil/pt/home/idh0/conceitos/o-que-e-o-idhm.html.

Rodrigues, F. F. L. (2015). Tendência de internações hospitalares de pacientes com e sem o diagnóstico de diabetes mellitus submetidos à amputação não traumática de extremidades inferiores, Ribeirão Preto - SP, 2001-2008. Tese de Doutorado, Escola de Enfermagem de Ribeirão Preto, Universidade de São Paulo, Ribeirão Preto. doi:10.11606/T.22.2015.tde-29052015-192858. Recuperado de www.teses.usp.br.

Rolim, D., Sampaio, S., Gonçalves-Dias, P., Almeida, P., Almeida-Lopes, J., \& Teixeira, J. F. (2015). Mortalidade depois da Amputação. Angiol Cir Vasc. 11(3), 166-170. https://doi.org/10.1016/j.ancv.2015.06.001

Santos, K. P. B., Luz, S. C. T., Mochizuki, L., \& d'Orsi, E. (2018). Burden of disease from lower limb amputations attributable to diabetes mellitus in Santa Catarina State, Brazil, 2008-2013. Cad. Saúde Pública. 34(1), e00013116. https://doi.org/10.1590/0102-311X00013116.

Senefonte, F. R. A., Rosa, G. R. P. S., Comparin, M. L., Covre, M. R., Jafar, M. B., Andrade, F. A. M., \& Nogueira Neto, E. (2012). Primary amputation in trauma: a profile of hospital Center-west region of Brazil. J Vasc Bras, 11(4). https://doi.org/10.1590/S1677-54492012000400004.

Sociedade Brasileira de Diabetes (2019). Sociedade Brasileira de Diabetes (SBD). Diretrizes da Sociedade Brasileira de Diabetes 2019-2020. Rio de Janeiro: Clannad Editora Científica. http://www.saude.ba.gov.br/wp-content/uploads/2020/02/Diretrizes-Sociedade-Brasileira-de-Diabetes-2019-2020.pdf.

Toscano, C. M., Sugita, T. H., Rosa, M., Pedrosa, H. C., Rosa, R., \& Bahia, L. R. (2018). Annual Direct Medical Costs of Diabetic Foot Disease in Brazil: A Cost of Illness Study. International journal of environmental research and public health, 15(1), 89. https://doi.org/10.3390/ijerph15010089.

World Health Organization (2014). Global status report on noncommunicable diseases 2014. WHO Document Production: Geneva, Switzerland. 